\title{
Elevated Plasma Level of 8-Hydroxy-2'-deoxyguanosine Is Associated with Primary Open-Angle Glaucoma
}

\author{
Altaf A. Kondkar $\left(D,{ }^{1,2}\right.$ Taif A. Azad ${ }^{1},{ }^{1}$ Tahira Sultan $\left(D,{ }^{1}\right.$ Essam A. Osman, \\ Faisal A. Almobarak $\mathbb{D}^{1,2}$ and Saleh A. Al-Obeidan ${ }^{1,2}$ \\ ${ }^{1}$ Department of Ophthalmology, College of Medicine, King Saud University, Riyadh, Saudi Arabia \\ ${ }^{2}$ Glaucoma Research Chair in Ophthalmology, College of Medicine, King Saud University, Riyadh, Saudi Arabia
}

Correspondence should be addressed to Altaf A. Kondkar; akondkar@gmail.com

Received 27 October 2019; Revised 29 March 2020; Accepted 10 April 2020; Published 27 April 2020

Academic Editor: Tomasz Zarnowski

Copyright (c) 2020 Altaf A. Kondkar et al. This is an open access article distributed under the Creative Commons Attribution License, which permits unrestricted use, distribution, and reproduction in any medium, provided the original work is properly cited.

Purpose. To determine the association between plasma 8-hydroxy-2'-deoxyguanosine (8-OHdG) levels, a marker for oxidative DNA damage, and patients with primary open-angle glaucoma (POAG) or its clinical phenotypes. Furthermore, we also examined the utility of plasma 8-OHdG as a potential biomarker in POAG. Materials and Methods. In a retrospective case-control study, plasma samples were obtained from 50 POAG cases and 45 glaucoma-free controls matched for age, sex, and ethnicity. 8-OHdG levels were measured in duplicate using an enzyme-linked immunosorbent assay (ELISA) on an automated ELISA analyzer. Results. There was no significant difference in age, sex, and systemic disease distribution between POAG cases and controls. Both mean and median 8-OHdG levels were significantly elevated in POAG cases and male subjects. The area under the receiver operating characteristic (ROC) curve value for plasma $8-\mathrm{OHdG}$ was $0.653(95 \%$ confidence interval $=0.54-0.76, p=0.010)$. The cutoff values based on quartile distribution and ROC curve analysis showed that elevated plasma 8-OHdG significantly increased the risk of POAG by more than 4 -folds. Plasma 8 -OHdG had a sensitivity of $78 \%$ and specificity of $53 \%$. In logistic regression analysis, 8 -OHdG showed a significant effect on POAG outcome $(p=0.016)$ independent of age, sex, smoking, and systemic diseases. However, no significant correlation was observed between 8-OHdG and specific clinical markers of glaucoma such as intraocular pressure $(p=0.699)$, cup/disc ratio $(p=0.213)$, and the number of antiglaucoma medications $(p=0.603)$. Conclusion. The study shows that there is a significant association between elevated plasma 8-OHdG and POAG, supporting the role of systemic oxidative stress-induced DNA damage in POAG pathogenesis. However, with a high rate of false-positivity, plasma 8OHdG may lack the ability to serve as a potential biomarker in POAG. Further studies in a much larger cohort are needed to confirm these findings.

\section{Introduction}

Glaucoma is a multifactorial and complex neurodegenerative disease that is caused by gradual apoptosis of retinal ganglion cells (RGCs) and the optic nerve head leading to irreversible blindness [1]. One of the pathologic mechanisms that may trigger apoptosis is oxidative stress via mitochondrial or endothelial damage, inflammation, and hypoxia [2]. Oxidative stress is believed to be majorly responsible for inducing molecular damage in the anterior chamber of the eye that may ultimately result in increased intraocular pressure (IOP) and subsequent manifestation of glaucoma [2].

The oxidative stress is generally induced by excessive generation of reactive oxygen species (ROS), mitochondrial dysfunction, impaired antioxidative defense mechanism, or a combination of these systems [2]. Under normal physiological conditions, there exists a balance between ROS production and clearance. However, excessive production of ROS in the cells may induce oxidative damage in the DNA, RNA, mitochondria, and other biomolecules, resulting in impairment of their cellular function(s) or cell death $[2,3]$. 
Cell-death induced by increased oxidative stress and ROS is involved in the pathogenesis of several neurodegenerative disorders such as Alzheimer, Parkinson, prion disease, and glaucoma $[4,5]$. Primary open-angle glaucoma (POAG) is an age-related disorder in which the trabecular meshwork (TM) malfunction plays a critical role [1]. In vivo studies in humans have demonstrated significantly more pronounced oxidative DNA damage in the TM cells of patients with glaucoma [6]. Furthermore, high IOP and visual field damage were both substantially proportional to the amount of oxidative DNA damage in the TM cells [7]. Besides, we have previously shown that plasma levels of total antioxidant status (TAS) were significantly lower in POAG patients as compared to nonglaucoma controls, thus supporting the role of oxidative stress-based mechanism in the pathogenesis of POAG [8]. Oxidative stress via ROS generation can induce breaks or base modifications in the DNA leading to the formation of DNA oxidation products such as 8-hydroxy-2' deoxyguanosine (8-OHdG) [9]. 8-OHdG is considered to be a reliable marker of oxidative DNA damage that can be easily quantified.

Based on the role of oxidative stress in the pathogenesis of glaucoma and our recent findings of elevated plasma 8OHdG levels in patients with pseudoexfoliation glaucoma (PXG) [10], we performed a case-control study to investigate the role of systemic oxidative stress-induced DNA damage in POAG. We examined an association between plasma 8$\mathrm{OHdG}$, as a marker of oxidative DNA damage, and POAG or its related clinical phenotypes. Besides, due to lack of any blood-based biomarker to assess the disease risk, we also examined the utility of plasma $8-\mathrm{OHdG}$ as a potential biomarker in POAG.

\section{Materials and Methods}

2.1. Study Population. The study adhered to the Declaration of Helsinki principles and was approved by the institutional review board committee at the College of Medicine, King Saud University, Riyadh, Saudi Arabia (approval number: 08-657). Participants of Saudi origin with the established clinical diagnosis of POAG $(n=50)$ and healthy controls $(n=45)$ were recruited at King Abdul-Aziz University Hospital in Riyadh, Saudi Arabia, following a written informed consent. Our team consists of 3 glaucoma consultants who carry out the measurements, review the data, and perform the diagnosis. All patients underwent standardized ophthalmic examination that included intraocular pressure (IOP) measurement by Goldmann applanation tonometry mounted at the slit lamp, anterior chamber angles examination by gonioscopy, dilated pupil examination of the lens and fundus, and visual field examination by Humphrey automated field analyzer. Patients were eligible for inclusion if they met the following clinical criteria for POAG: adultonset of the disease; intraocular pressure (IOP) $\geq 21 \mathrm{mmHg}$ in one or both eyes before initiation of glaucoma treatment; normal-appearing, bilaterally open anterior chamber angles by gonioscopy; optic nerve appearance characteristic of the optic discs typically observed in POAG (with localized narrowing or absence of the neuro-retinal rim, with the amount of cupping exceeding the amount of pallor of the rim, and with asymmetric cupping of the optic discs); and corresponding visual field (Humphrey Field Analyzer II, Carl Zeiss Meditec, Inc., Dublin, CA, USA; using a full threshold 24-2 program) abnormalities typical of glaucoma [11]. The exclusion criteria included historical, clinical, or biochemical evidence of another possible optic neuropathic process affecting either eye, significant visual loss in both eyes not associated with glaucoma, evidence of secondary glaucoma, e.g., pigmentary dispersion syndrome, pseudoexfoliation, history of ocular trauma, steroid usage or any antioxidant supplements and vitamins, any infectious or immunomodulating diseases (rheumatoid arthritis, lupus, Crohn's disease), or refusal to participate.

Among antiglaucoma medications, beta-blockers (e.g., timolol), prostaglandin analogue (e.g., latanoprost), and carbonic anhydrase inhibitors (e.g., acetazolamide) were the most common drugs prescribed to the glaucoma patients.

The ethnically matched control group included subjects with age $>20$ years at recruitment; normal IOP $(<21 \mathrm{mmHg})$; normal optic disk with open anterior chamber angles on examination; and no history of ocular disease(s) or eye surgeries. Other information related to the history of systemic diseases, family history, and smoking status was obtained from medical records or personal interviews. Subjects taking any antioxidant supplements, vitamins, or having any infectious or immunomodulating diseases (rheumatoid arthritis, lupus, and Crohn's disease), or refusing to participate were excluded.

2.2. Levels of Plasma 8-Hydroxy 2'-deoxyguanosine. Estimation of 8-OHdG levels from plasma was performed using a commercial competitive sandwich enzyme-linked immunosorbent assay (ELISA) kit (Trevigen, Gaithersburg, MD, USA). The assay was performed in duplicate as per the manufacturer's instructions on an automated ELISA analyzer, ChemWell-T (Awareness Technology Inc., FL, USA). The concentrations of 8-OHdG levels were determined from a standard curve and expressed in $\mathrm{ng} / \mathrm{mL}$.

2.3. Statistical Analysis. Statistical analysis was performed with SPSS version 19.0 (IBM Corp., Armonk, New York, USA) and Stat View software version 5.0 (SAS Institute, Cary, NC, USA). Data were tabulated as mean, median, frequency, and percentages. Normality testing for 8-OHdG levels was done using Kolmogorov-Smirnov test. Mean differences between groups were tested by independent sample $t$-test. Mann-Whitney $U$ test was used to compare median values between the patients and controls. The categorical variables were tested by Chi-square test. The associated risk was expressed as odds ratio (OR) and 95\% confidence interval (CI). The correlation testing was done using Spearman's method. A binary logistic regression analysis was performed to estimate the impact and effect of mean 8-OHdG level and other risk factors on disease outcome. To determine clinical utility of $8-\mathrm{OHdG}$, area under the receiver operating characteristic (ROC) curve (AUC), sensitivity (true-positive), and specificity (true-negative) of 
the assay were examined by ROC curve and analyzed by Mann-Whitney test. All statistical tests were two-sided, and $p$ value $<0.05$ was considered statistically significant.

\section{Results}

3.1. Study Population and Plasma 8-OHdG Levels. As shown in Table 1, the study groups showed no significant difference in terms of age, gender, smoking habits, and other systemic disease conditions $(p>0.05)$. The prevalence of family history of glaucoma was significantly more in the patient group $(p=0.012)$. Besides, the mean 8 -OHdG level was significantly elevated in the POAG patients $(22.61 \pm 12.05 \mathrm{ng} / \mathrm{mL})$ than the controls $(16.95 \pm 10.66 \mathrm{ng} /$ $\mathrm{mL} ; p=0.018)$. Also, the median 8 -OHdG levels were significantly high $(p=0.010)$ between controls $(13.78 \mathrm{ng} /$ $\mathrm{mL})$ and POAG $(18.53 \mathrm{pg} / \mathrm{mL})$. Similarly, gender-stratification showed that both the mean and median levels of 8OHdG were significantly elevated in POAG males but not in females (Table 1). Figure 1 shows the box-plot distribution of plasma 8-OHdG based on sample types and gender in POAG cases and controls. The clinical characteristics of POAG patients are shown in Table 2, and a representative imaging data of a POAG patient is shown in Supplementary Figure 1.

3.2. Plasma 8-OHdG and Risk of POAG. To evaluate the risk of POAG at different levels of 8-OHdG (dose-related trend), the uncategorized data (not as cases and controls) were dichotomized into quartiles to identify two cutoff values of $11.18 \mathrm{ng} / \mathrm{mL}$ (first quartile or the $25^{\text {th }}$ percentile) and 26.60 (third quartile or the $75^{\text {th }}$ percentile). Subjects were then categorized into three groups by using these two cutoff values as shown in Table 3. Quartile distribution data showed that there was a significant additive effect of increasing levels of 8 -OHdG on POAG outcome $\left(\chi^{2}=8.58\right.$, $\mathrm{df}=2 ; p=0.014)$. Besides, compared to $8-\mathrm{OHdG}$, levels less than the first quartile or the $25^{\text {th }}$ percentile $(<11.18 \mathrm{ng} / \mathrm{mL})$, subjects with interquartile $\left(25^{\text {th }}-75^{\text {th }}\right.$ percentile) levels, and those higher than the third quartile $\left(75^{\text {th }}\right.$ percentile) exhibited a significantly increased risk of POAG. Similarly, the ROC curve also allows identification of best cutoff values to maximize sensitivity and specificity [12]. Based on ROC curve analysis, a cutoff value of $14.8 \mathrm{ng} / \mathrm{mL}$ also exhibited 4 fold increased risk of POAG $(p=0.002)$ (Table 2).

3.3. 8-OHdG as a Biomarker. ROC curve was generated to evaluate the potential of plasma 8-OHdG to discriminate between POAG cases and controls (Figure 2). The analysis yielded a significant $(p=0.010)$ AUC value of $0.653(95 \%$ $\mathrm{CI}=0.541-0.765)$. The accuracy of a test marker is directly proportional to the AUC value which can vary between 0.5 and 1.0 , with 0.5 indicating poor accuracy, $0.5-0.7$, moderate, $0.7-0.9$, highly accurate, and 1.0 suggesting a best-fit, thus indicating that plasma $8-\mathrm{OHdG}$ was a significant moderate discriminator between POAG cases and controls. The sensitivity, specificity, and other characteristics of 8OHdG as a biomarker based on the ROC curve threshold are shown in Table 4. At a cutoff value of $14.8 \mathrm{ng} / \mathrm{mL}$, plasma 8OHdG had a sensitivity of $78 \%$ and specificity of $53 \%$.

3.4. Plasma 8-OHdG and Other Potential Confounders. A binary logistic regression analysis was performed to investigate the effect of multiple factors such as age, sex, systemic diseases, smoking, and plasma 8-OHdG levels in patients with POAG, using diseased/nondiseased as a dependent variable (outcome). Except for plasma 8-OHdG ( $p=0.016$ ), none of the other confounding variables showed any significant effect on the disease outcome (Table 5).

3.5. Correlation between 8-OHdG and Glaucoma Indices in POAG Patients. There was no significant correlation between 8-OHdG and age, sex, and other clinical phenotypic markers of disease such as IOP, cup/disc ratio, and the number of antiglaucoma medication (Table 6).

\section{Discussion}

Both endogenous (normal cellular metabolism) and exogenous factors (e.g., UV) can generate ROS, which as a result of impaired pro-oxidant and antioxidant balance, can cause DNA damage and contribute significantly to glaucomatous neurodegeneration [13]. Base guanine is considered to be highly susceptible to oxidative modifications due to its low redox potential, and its most common byproduct $8-\mathrm{OHdG}$ is believed to be an excellent marker for oxidative stress-induced DNA damage [14]. Oxidative DNA damage constitutes a significant threat to genetic integrity and thus has been implicated in the pathogenesis of complex human diseases such as cancer and neurodegenerative disorders, including glaucoma $[2,4,15,16]$. In this study, we report an association between increased levels of systemic 8-OHdG, a marker of oxidative stress-induced DNA damage, and POAG.

Several studies in the past have provided a vital link between increased levels of 8-OHdG and glaucomatous optic neuropathy $[6,7,17]$. Early studies by Izzotti et al. demonstrated a more than 3 -fold increase in 8 -OHdG levels in the TM cells of glaucoma patients with the amount of oxidative DNA damage correlating to IOP increase and visual filed loss, thereby providing convincing evidence for the role of oxidative DNA damage in glaucoma [6, 7]. Since then, studies in human samples have also consistently reported an increase in 8-OHdG levels in different forms of glaucoma. In a small study consisting of POAG and PXG, both aqueous humor $(\mathrm{AH})$ and serum samples were elevated in cases compared to controls [17]. Similarly, Mohanty and colleagues reported that both plasma and $\mathrm{AH}$ 8-OHdG were elevated in POAG patients as compared to cataract controls [18]. Moreover, the increase was attributed to reduced expression of DNA repair enzymes of the base excision repair pathway [18], and a strong positive correlation between plasma 8-OHdG levels and AH 8-OHdG levels were reported, suggesting that systemic 8-OHdG levels could be predictive of local 8-OHdG levels in the eye [18]. In another recent study by Mumcu et al. increased 8-OHdG levels, as 
TABle 1: Demographic, systemic disease status, and 8-hydroxy-2'-deoxyguanosine levels between patients with primary open-angle glaucoma and healthy controls.

\begin{tabular}{|c|c|c|c|}
\hline Variables & Controls $(n=45)$ & POAG $(n=50)$ & $p$ value \\
\hline Age in years, mean (SD) & $59.9(8.3)$ & $62.3(9.2)$ & $0.191^{\mathrm{a}}$ \\
\hline Male/female, $n$ & $30 / 15$ & $28 / 22$ & $0.287^{\mathrm{b}}$ \\
\hline \multicolumn{4}{|l|}{ Systemic diseases, $n(\%)$} \\
\hline Diabetes mellitus & $6(13.3)$ & $8(16.0)$ & $0.714^{\mathrm{b}}$ \\
\hline Hypertension & $5(11.1)$ & $7(14.0)$ & $0.672^{\mathrm{b}}$ \\
\hline Coronary artery disease & $2(4.4)$ & $4(8.0)$ & $0.477^{\mathrm{b}}$ \\
\hline Hypercholesterolemia & $2(4.4)$ & $3(6.0)$ & $0.735^{\mathrm{b}}$ \\
\hline Family history of glaucoma & $1(2.2)$ & $9(18.0)$ & $0.012^{\mathrm{b}}$ \\
\hline Smokers & $5(11.1)$ & $7(14.0)$ & $0.672^{\mathrm{b}}$ \\
\hline \multicolumn{4}{|l|}{ 8-OHdG levels, $\mathrm{ng} / \mathrm{mL}$} \\
\hline Mean (SD) & $16.95(10.66)$ & $22.61(12.05)$ & $0.018^{\mathrm{a}}$ \\
\hline Median & 13.78 & 18.35 & $0.010^{\mathrm{c}}$ \\
\hline \multicolumn{4}{|l|}{ Males } \\
\hline Mean (SD) & $17.02(11.02)$ & $23.15(12.24)$ & $0.049^{\mathrm{a}}$ \\
\hline Median & 13.12 & 18.08 & $0.024^{\mathrm{c}}$ \\
\hline \multicolumn{4}{|l|}{ Females } \\
\hline Mean (SD) & $16.80(10.29)$ & $21.92(12.06)$ & $0.188^{\mathrm{a}}$ \\
\hline Median & 17.97 & 19.24 & $0.254^{\mathrm{c}}$ \\
\hline
\end{tabular}

Note. ${ }^{\mathrm{a}}$ Independent sample $t$-test (two-tailed); ${ }^{\mathrm{b}} \mathrm{Chi}^{2}$ test; ${ }^{\mathrm{c}} \mathrm{Mann}$-Whitney $U$ test. 8-OHdG, 8-hydroxy-2'-deoxyguanosine; POAG, primary open-angle glaucoma.

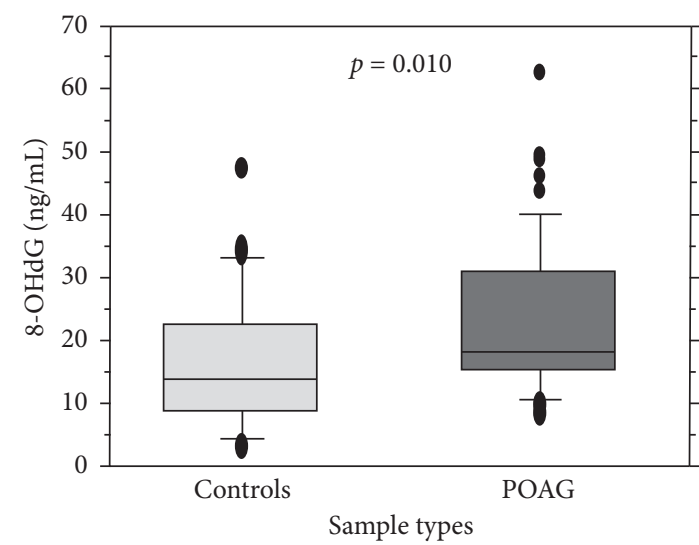

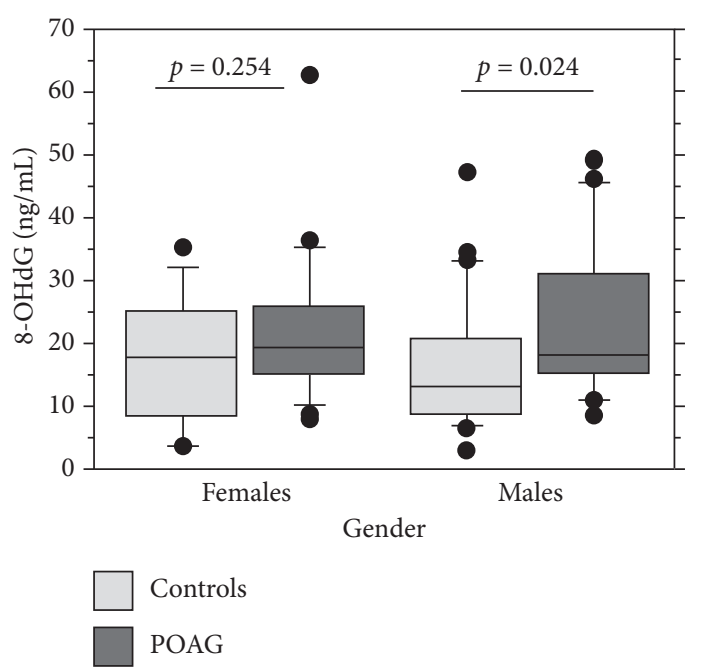

(b)

Figure 1: Box plot showing the distribution of 8-hydroxy-2'-deoxyguanosine levels: (a) between primary open-angle glaucoma cases and controls and (b) according to gender in cases and controls. 8-OHdG, 8-hydroxy-2'-deoxyguanosine; POAG, primary open-angle glaucoma.

measured by high-performance liquid chromatography, and decreased paraoxonase-1 activity were associated with POAG [19]. Likewise, studies by Yuki et al. have reported increased urinary 8-OHdG levels to be associated with glaucomatous visual field progression in subjects with normal tension glaucoma [20,21], and Chang and colleagues have reported elevated serum $8-\mathrm{OHdG}$ in patients with primary angle-closure glaucoma of Chinese origin [22]. Besides, 8-OHdG levels have also been reported as potential diagnostic marker in other neurodegenerative diseases such as dementia (e.g., Alzheimer's disease) and Parkinson's disease (PD). In an excellent study by Choromanska and colleagues, significantly increased oxidative damage (measured as 8-OHdG among others) and decreased antioxidant
TABLe 2: Clinical characteristics of primary open-angle glaucoma patients.

\begin{tabular}{lc}
\hline Characteristics & POAG \\
\hline Age in years, mean (SD) & $62.3(9.2)$ \\
Male/female, $n$ & $28 / 22$ \\
${ }^{a}$ Intraocular pressure mmHg, mean (SD) & $23.8(8.0)$ \\
Cup/disc ratio & $0.78(0.15)$ \\
Number of antiglaucoma medications, mean (SD) & $1.98(0.90)$
\end{tabular}

${ }^{\mathrm{a} B a s e l i n e}$ corrected for central corneal thickness. POAG, primary openangle glaucoma.

status were reported in stimulated and nonstimulated saliva. The results of the study suggested that changes in salivary redox homeostasis are independent of systemic (plasma/ 
TABLE 3: Plasma levels of 8-hydroxy-2'-deoxyguanosine and the risk of primary open-angle glaucoma.

\begin{tabular}{|c|c|c|c|c|}
\hline 8-OHdG cutoff ng/mL & Controls no. (\%) & POAG no. (\%) & Odds ratio ( $95 \%$ confidence interval) & $p$ value $^{\mathrm{a}}$ \\
\hline \multicolumn{5}{|l|}{ By quartiles $^{\dagger *}$} \\
\hline$<11.18$ & $17(33.3)$ & $6(12.0)$ & Reference & - \\
\hline $11.18-26.60$ & $19(26.6)$ & $30(60.0)$ & $4.47(1.49-13.35)$ & 0.005 \\
\hline$>26.60$ & $9(40.0)$ & $14(28.0)$ & $4.40(1.26-15.41)$ & 0.017 \\
\hline \multicolumn{5}{|l|}{ By ROC curve } \\
\hline$<14.80$ & $24(53.3)$ & $11(22.0)$ & Reference & \\
\hline$\geq 14.80$ & $21(46.6)$ & $39(78.0)$ & $4.05(1.66-9.86)$ & 0.002 \\
\hline
\end{tabular}

Note. ${ }^{\text {a }}$ Pearson's $\mathrm{Chi}^{2}$ test; ${ }^{\dagger}$ first quartile $\left(<25^{\text {th }}\right.$ percentile $)$; interquartile $\left(25^{\text {th }}-75^{\text {th }}\right.$ percentile $)$; third quartile $\left(>75^{\text {th }}\right.$ percentile). ${ }^{*}$ Overall $\mathrm{Chi}{ }^{2}=8.58, \mathrm{df}=2$, $p<0.014$. 8-OHdG, 8-hydroxy-2'-deoxyguanosine; POAG, primary open-angle glaucoma; ROC, receiver operator characteristic.

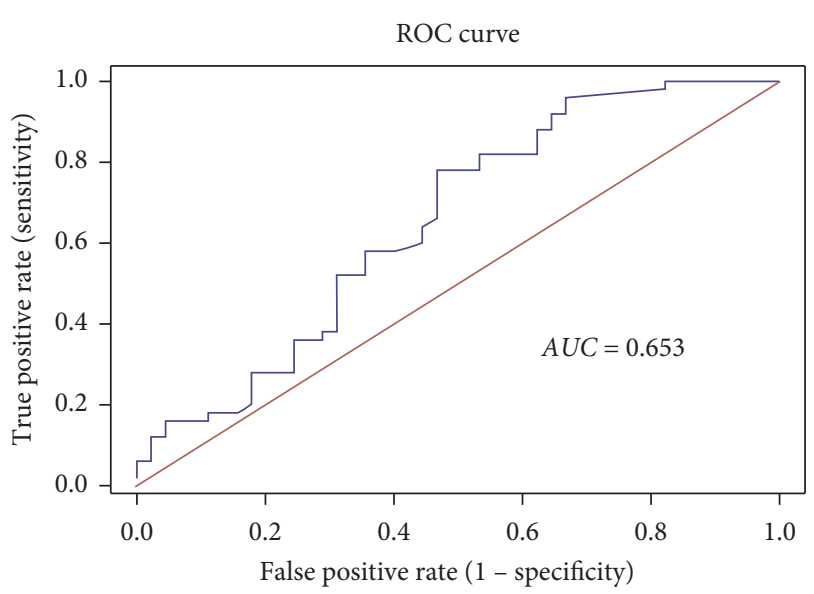

FIgURE 2: ROC curve analysis. AUC, area under the receiver operator characteristic curve; ROC, receiver operator characteristic.

erythrocytes) changes in the course of dementia [23]. Likewise, cerebrospinal fluid 8-OHdG levels were significantly increased in nondemented PD patients suggesting that 8 -OHdG levels could potentially complement neurochemically supported diagnosis of PD [24].

In concordance with these reports, our findings show that both the mean and median levels of $8-\mathrm{OHdG}$ are significantly increased in our POAG cohort and increase the risk of POAG by more than 4 -folds. However, plasma 8OHdG showed no significant correlation with IOP, cup/disc ratio, and number of antiglaucoma medication, indicating a lack of association with these clinical phenotypes or markers used to assess disease severity. Furthermore, the ROC curve analysis showed that plasma $8-\mathrm{OHdG}$, as a biomarker, has good sensitivity but low specificity. This indicates that plasma 8-OHdG may be able to predict disease (POAG) with a high rate of false-positivity and thus may not serve as a potential circulating biomarker in POAG. It is noteworthy that since 8-OHdG is an oxidative stress biomarker, its levels can be influenced by aging, infectious diseases, smoking habits, and other systemic diseases such as diabetes, hypertension, or coronary disease [3, 25-27]. Thus, care was taken to exclude individuals with infectious or autoimmune diseases or those taking dietary vitamins and supplements. Besides, the control group showed no significant deviation than the patient group in terms of age, ethnicity, gender, smoking habits, and systemic disease status. Furthermore,
TABLE 4: ROC curve characteristics of plasma 8-hydroxy-2'deoxyguanosine as a biomarker in POAG

\begin{tabular}{lcc}
\hline Characteristics & Value & $95 \%$ confidence interval \\
\hline Cutoff value, ng/mL & $\geq 14.8$ & - \\
AUC (SE) & $0.653(0.057)$ & $0.54-0.76$ \\
$p$ value & 0.010 & - \\
$\begin{array}{l}\text { Sensitivity, \% } \\
\text { Specificity, \% }\end{array}$ & 78.0 & $64.0-88.4$ \\
$\begin{array}{l}\text { Positive likelihood } \\
\text { ratio }\end{array}$ & 53.3 & $37.8-68.3$ \\
$\begin{array}{l}\text { Negative likelihood } \\
\text { ratio }\end{array}$ & 1.67 & $1.18-2.36$ \\
$\begin{array}{l}\text { Positive predictive } \\
\text { value, \% }\end{array}$ & 0.41 & $0.23-0.74$ \\
$\begin{array}{l}\text { Negative predictive } \\
\text { value, \% }\end{array}$ & 65.0 & $56.8-72.4$ \\
Accuracy, \% & 68.5 & $54.7-79.7$ \\
\hline
\end{tabular}

AUC, area under the receiving operating characteristics curve; POAG, primary open-angle glaucoma; ROC, receiver operating characteristic.

logistic regression analysis indicated that the significant effect of plasma 8-OHdG on POAG risk was independent of age, gender, smoking, or systemic diseases, thereby suggesting that elevated plasma 8 -OHdG could be more plausibly related to POAG condition rather than to any of these confounding variables. Clearly, further investigations are needed in a much larger cohort to confirm these findings. However, the observed increase in the plasma levels of 8OHdG clearly suggests an increased oxidative stress-induced DNA damage and its plausible association with glaucomatous degeneration and is in agreement with our previous observation in PXG patients of Saudi origin [10].

The role of 8-OHdG in glaucoma development and progression is still speculative. Local increase of oxidative DNA damage in the eye (as shown in TM cells) [6] can cause TM degeneration, hypoxia, and rise in IOP. These can lead to the clinical onset of glaucoma [16]. Besides, failure to repair DNA lesions can result in transversion mutations which can have serious biological consequences [28]. Oxidative DNA damage may accelerate telomere shortening and cause cell senescence associated with aging and degenerative diseases $[28,29]$; or oxidative stress through the formation of 8OHdG may induce epigenetic instability which can activate oncogenes or inactive tumor suppressor genes $[15,28]$. The pathophysiological effects of oxidative stress and ROS in glaucoma through glial cell damage, autophagy, nuclearkappa B activation (signaling), nitrite stress, and alterations 
TABLE 5: Binary logistic regression analysis to assess the effect of 8hydroxy-2' -deoxyguanosine levels and other potential confounders on disease outcome.

\begin{tabular}{lcccc}
\hline Variables & B & SE & $\begin{array}{c}\text { Odds ratio } \\
(95 \% \mathrm{CI})\end{array}$ & $p$ value \\
\hline Age & 0.039 & 0.025 & $1.04(0.99-1.09)$ & 0.120 \\
Sex $^{\mathrm{a}}$ & -0.569 & 0.449 & $0.56(0.23-1.36)$ & 0.205 \\
Diabetes & 0.139 & 0.715 & $1.15(0.28-4.66)$ & 0.846 \\
Hypertension & 0.066 & 0.804 & $1.06(0.22-5.16)$ & 0.935 \\
Heart disease & -0.009 & 1.060 & $0.99(0.12-7.9)$ & 0.994 \\
Hypercholesterolemia & -0.633 & 1.105 & $0.53(0.06-4.63)$ & 0.567 \\
Smoking & 0.557 & 0.727 & $1.74(0.42-7.25)$ & 0.443 \\
8-OHdG & 0.052 & 0.022 & $1.05(1.01-1.10)$ & 0.016 \\
Constant & -2.79 & 1.662 & 0.06 & 0.092 \\
\hline
\end{tabular}

${ }^{\mathrm{a}}$ Females as reference. 8-OHdG, 8-hydroxy-2'-deoxyguanosine.

TABle 6: Correlation analysis between 8-hydroxy-2'-deoxyguanosine levels and glaucoma specific clinical indices in patients.

\begin{tabular}{lcc}
\hline Variables & $R$ & $p$ value \\
\hline Age & -0.067 & 0.643 \\
Sex & 0.008 & 0.953 \\
Intraocular pressure, mmHg & 0.056 & 0.699 \\
Cup/disc ratio & -0.179 & 0.213 \\
Number of antiglaucoma medications & 0.075 & 0.603 \\
\hline
\end{tabular}

$R$, Spearman's correlation coefficient.

in ocular hemodynamics to propagate inflammation and optic nerve damage or RGC death are also well documented (as reviewed elsewhere) [2]. Oxidative stress has also been strongly linked to mitochondrial abnormalities and reduced total antioxidant status as has been consistently proven in POAG $[8,30]$ and PXG $[31,32]$, thereby substantiating our current finding that oxidative stress-induced DNA damage may have a significant role to play in the pathogenesis of POAG and that the high levels of $8-\mathrm{OHdG}$ observed in our study may plausibly contribute to POAG pathogenesis by similar mechanism(s). Paradoxically, exogenous (synthetic) 8-OHdG has been suggested as a potential candidate for the treatment and prevention of inflammation-based gastrointestinal diseases and cancer [33].

The study has certain limitations. The systemic increase in 8-OHdG may not reflect the actual microenvironment of the cells/tissues in the anterior chamber of the eye which are continuously exposed to ROS and are more related to the disease. To achieve this, analysis of AH samples would be appropriate. Besides, the study is purely descriptive with no mechanistic evidence to suggest causal implications of high 8-OHdG in POAG. Lastly, the study is limited in its capacity to evaluate $8-\mathrm{OHdG}$ association with disease severity or advancement due to its relatively small sample size for subgroup analyses and low number of patient samples in the lowest-quartile of 8-OHdG. This may also explain the lack of correlation of plasma $8-\mathrm{OHdG}$ with IOP and cup/disc ratio; or its lack of association in females (subgroup). An investigation in a large population-based cohort would certainly confirm these findings. However, based on the data observed in this study, an actual mean difference of $6 \mathrm{ng} / \mathrm{mL}$ in the POAG and controls with a standard deviation of 10 , the probability (power) to detect an association with POAG is $>0.8$. The type I error probability associated with this test for the null hypothesis is 0.05 .

\section{Conclusion}

In conclusion, the study provides an evidence for a significant association between plasma $8-\mathrm{OHdG}$ and POAG and suggests that elevated levels of systemic $8-\mathrm{OHdG}$ may be a significant risk factor for POAG. The findings add to the growing body of evidence supporting the role of systemic oxidative stressinduced DNA damage in the development or progression of POAG. However, plasma $8-\mathrm{OHdG}$ did not show any significant correlation with clinical markers of POAG (e.g., IOP and cup/disc ratio) but showed moderate ability to discriminate between cases and controls, and exhibited a high rate of false-positivity. Thus, plasma 8-OHdG may not serve as a potential clinical biomarker in POAG. Further investigations in a much larger cohort are needed to validate these results and assess the association/correlation between systemic oxidative DNA damage status, glaucoma severity, and extent of visual field damage in POAG.

\section{Data Availability}

The data supporting the conclusions of this article are all presented within the report.

\section{Ethical Approval}

The study adhered to the tenets of the Declaration of Helsinki and had received approval from the Institutional Review Board and Research Ethics Committee of College of Medicine, King Saud University, Riyadh, Saudi Arabia (approval \#08-657).

\section{Consent}

A written informed consent was obtained from all participants before their inclusion in this study.

\section{Disclosure}

The authors alone are responsible for the content and writing of the paper.

\section{Conflicts of Interest}

The authors declare no conflicts of interest.

\section{Authors' Contributions}

All authors contributed toward data analysis, drafting, and revising the paper and agree to be accountable for all aspects of the work.

\section{Acknowledgments}

The authors would like to thank the Vice Deanship of Scientific Research Chair, Glaucoma Research Chair in Ophthalmology at the King Saud University. This work was 
supported by King Saud University through Vice Deanship of Scientific Research Chair, Glaucoma Research Chair in Ophthalmology, but had no role in the design of the study and collection, analysis, and interpretation of data and in writing the manuscript.

\section{Supplementary Materials}

Supplementary Figure 1: (a) optic nerve with advanced damage and (b) corresponding advanced visual field defect with HVF, 24-2 strategy in a POAG patient. (Supplementary Materials)

\section{References}

[1] R. N. Weinreb, T. Aung, and F. A. Medeiros, "The pathophysiology and treatment of glaucoma," JAMA, vol. 311, no. 18, pp. 1901-1911, 2014.

[2] M. Nita and A. Grzybowski, "The role of the reactive oxygen species and oxidative stress in the pathomechanism of the agerelated ocular diseases and other pathologies of the anterior and posterior eye segments in adults," Oxidative Medicine and Cellular Longevity, vol. 2016, Article ID 3164734, 23 pages, 2016.

[3] M. S. Cooke, M. D. Evans, M. Dizdaroglu, and J. Lunec, "Oxidative DNA damage: mechanisms, mutation, and disease," The FASEB Journal, vol. 17, no. 10, pp. 1195-1214, 2003.

[4] Z. Liu, T. Zhou, A. C. Ziegler, P. Dimitrion, and L. Zuo, "Oxidative stress in neurodegenerative diseases: from molecular mechanisms to clinical applications," Oxidative Medicine and Cellular Longevity, vol. 2017, no. 4, Article ID 2525967, 11 pages, 2017.

[5] G. Tezel, "Oxidative stress in glaucomatous neurodegeneration: mechanisms and consequences," Progress in Retinal and Eye Research, vol. 25, no. 5, pp. 490-513, 2006.

[6] A. Izzotti, C. Cartiglia, S. De Flora, and S. Saccà, "Methodology for evaluating oxidative DNA damage and metabolic genotypes in human trabecular meshwork," Toxicology Mechanisms and Methods, vol. 13, no. 3, pp. 161-168, 2003.

[7] S. C. Saccà, A. Pascotto, P. Camicione, P. Capris, and A. Izzotti, "Oxidative DNA damage in the human trabecular meshwork," Archives of Ophthalmology, vol. 123, no. 4, pp. 458-463, 2005.

[8] K. K. Abu-Amero, A. A. Kondkar, A. Mousa, E. A. Osman, and S. A. Al-Obeidan, "Decreased total antioxidants in patients with primary open angle glaucoma," Current Eye Research, vol. 38, no. 9, pp. 959-964, 2013.

[9] T. Hemnani and M. S. Parihar, "Reactive oxygen species and oxidative DNA damage," Indian Journal of Physiology and Pharmacology, vol. 42, no. 4, pp. 440-452, 1998.

[10] A. A. Kondkar, T. Sultan, T. A. Azad, L. Tabussum, E. A. Osman, and S. A. Al-Obeidan, "Increased plasma levels of 8-Hydroxy-2'-deoxyguanosine (8-OHdG) in patients with pseudoexfoliation glaucoma," Journal of Ophthalmology, vol. 2019, Article ID 8319563, 7 pages, 2019.

[11] J. Kim, L. G. Dally, F. Ederer et al., "The Advanced Glaucoma Intervention Study (AGIS): 14. Distinguishing progression of glaucoma from visual field fluctuations," Ophthalmology, vol. 111, no. 11, pp. 2109-2116, 2004.

[12] J. Swets, "Measuring the accuracy of diagnostic systems," Science, vol. 240, no. 4857, pp. 1285-1293, 1988.

[13] J. Cadet and J. R. Wagner, "DNA base damage by reactive oxygen species, oxidizing agents, and UV radiation," Cold
Spring Harbor Perspectives in Biology, vol. 5, no. 2, Article ID a012559, 2013.

[14] A. Valavanidis, T. Vlachogianni, and C. Fiotakis, "8-hydroxy$2^{\prime}$-deoxyguanosine (8-OHdG): a critical biomarker of oxidative stress and carcinogenesis," Journal of Environmental Science and Health, Part C, vol. 27, no. 2, pp. 120-139, 2009.

[15] N. Nishida, T. Arizumi, M. Takita et al., "Reactive oxygen species induce epigenetic instability through the formation of 8-hydroxydeoxyguanosine in human hepatocarcinogenesis," Digestive Diseases, vol. 31, no. 5-6, pp. 459-466, 2013.

[16] A. Izzotti, A. Bagnis, and S. Sacca, "The role of oxidative stress in glaucoma," Mutation Research/Reviews in Mutation Research, vol. 612, no. 2, pp. 105-114, 2006.

[17] R. Sorkhabi, A. Ghorbanihaghjo, A. Javadzadeh, N. Rashtchizadeh, and M. Moharrery, "Oxidative DNA damage and total antioxidant status in glaucoma patients," Molecular Vision, vol. 17, pp. 41-46, 2011.

[18] K. Mohanty, R. Dada, and T. Dada, "Oxidative DNA damage and reduced expression of DNA repair genes: role in primary open angle glaucoma (POAG)," Ophthalmic Genetics, vol. 38, no. 5, pp. 446-450, 2017.

[19] U. Y. Mumcu, I. Kocer, O. Ates, and H. H. Alp, "Decreased paraoxonase1 activity and increased malondialdehyde and oxidative DNA damage levels in primary open angle glaucoma," International Journal of Ophthalmology, vol. 9, no. 10, pp. 1518-1520, 2016.

[20] K. Yuki and K. Tsubota, "Increased urinary 8-Hydroxy-2'deoxyguanosine $(8-\mathrm{OHdG}) /$ creatinine level is associated with the progression of normal-tension glaucoma," Current Eye Research, vol. 38, no. 9, pp. 983-988, 2013.

[21] K. Yuki, D. Murat, I. Kimura, and K. Tsubota, "Increased serum total antioxidant status and decreased urinary 8-hydroxy-2'-deoxyguanosine levels in patients with normaltension glaucoma," Acta Ophthalmologica, vol. 88, no. 7, pp. e259-e264, 2010.

[22] D. Chang, Q. Sha, X. Zhang et al., "The evaluation of the oxidative stress parameters in patients with primary angleclosure glaucoma," PLoS One, vol. 6, no. 11, p. e27218, 2011.

[23] M. Choromanska, A. Klimiuk, P. Kostecka-Sochon et al., "Antioxidant defence, oxidative stress and oxidative damage in saliva, plasma and erythrocytes of dementia patients. Can salivary AGE be a marker of dementia?" International Journal of Molecular Sciences, vol. 18, no. 10, 2017.

[24] K. Gmitterova, U. Heinemann, J. Gawinecka et al., "8-OHdG in cerebrospinal fluid as a marker of oxidative stress in various neurodegenerative diseases," Neurodegenerative Diseases, vol. 6, no. 5-6, pp. 263-269, 2009.

[25] I. Dalle-Donne, R. Rossi, R. Colombo, D. Giustarini, and A. Milzani, "Biomarkers of oxidative damage in human disease," Clinical Chemistry, vol. 52, no. 4, pp. 601-623, 2006.

[26] A. Di Minno, L. Turnu, B. Porro et al., "8-Hydroxy-2Deoxyguanosine levels and cardiovascular disease: a systematic review and meta-analysis of the literature," Antioxidants \& Redox Signaling, vol. 24, no. 10, pp. 548-555, 2016.

[27] Q. Y. Dong, Y. Cui, L. Chen, J. Song, and L. Sun, "Urinary 8hydroxydeoxyguanosine levels in diabetic retinopathy patients," European Journal of Ophthalmology, vol. 18, no. 1, pp. 94-98, 2008.

[28] E. Markkanen, "Not breathing is not an option: how to deal with oxidative DNA damage," DNA Repair, vol. 59, pp. 82105, 2017.

[29] S. Rizvi, S. T. Raza, and F. Mahdi, "Telomere length variations in aging and age-related diseases," Current Aging Science, vol. 7, no. 3, pp. 161-167, 2014. 
[30] K. K. Abu-Amero, J. Morales, and T. M. Bosley, "Mitochondrial abnormalities in patients with primary open-angle glaucoma," Investigative Opthalmology \& Visual Science, vol. 47, no. 6, pp. 2533-2541, 2006.

[31] K. K. Abu-Amero, T. M. Bosley, and J. Morales, "Analysis of nuclear and mitochondrial genes in patients with pseudoexfoliation glaucoma," Molecular Vision, vol. 14, pp. 29-36, 2008.

[32] K. K. Abu-Amero, A. A. Kondkar, A. Mousa, E. A. Osman, and S. A. Al-Obeidan, "Decreased total antioxidants status in the plasma of patients with pseudoexfoliation glaucoma," Molecular Vision, vol. 17, pp. 2769-2775, 2011.

[33] C.-Y. Ock, E. H. Kim, D. J. Choi, H. J. Lee, K. B. Hahm, and M. H. Chung, "8-Hydroxydeoxyguanosine: not mere biomarker for oxidative stress, but remedy for oxidative stressimplicated gastrointestinal diseases," World Journal of Gastroenterology, vol. 18, no. 4, pp. 302-308, 2012. 